\title{
REVIEW
}

\section{Intracranial Neurophysiological Correlates Related to the Processing of Faces}

\author{
M. Seeck,* C. M. Michel, ${ }^{+, \ddagger}$ O. Blanke, ${ }^{*}$ G. Thut, ${ }^{+, \ddagger}$ T. Landis, ${ }^{\ddagger}$ \\ and D. L. Schomer ${ }^{\S, 1}$ \\ "Laboratory of Presurgical Epilepsy Evaluation, Functional Neurology and Neurosurgery \\ Program of the University Hospitals Lausanne and Geneva, Switzerland; ${ }^{\dagger}$ Plurifaculty \\ Program of Cognitive Neuroscience and ${ }^{\ddagger}$ Department of Neurology, University Hospital \\ of Geneva, Geneva, Switzerland; and ${ }^{\S}$ Beth Israel Deaconess Medical Center, \\ Harvard University, Boston, Massachusetts 02215
}

Received J une 19, 2001; accepted for publication August 27, 2001

\begin{abstract}
Face perception and recognition is an intriguing ability, already present in neonates. Numerous studies in patients with brain lesions identified the temporo-occipital cortex as the crucial structure for this capacity. Analysis of electrical signals (EEG) inside the brain of patients implanted with intracranial electrodes for diagnostic purposes allows researchers to describe the temporal and spatial organization of responses to various aspects of face processing in human subjects. Several findings have emerged and appear relevant for cerebral organization in general: (1) Selective face responses were obtained from the basal temporo-occipital cortex at around $200 \mathrm{~ms}$ (N200); however, other structures such as the lateral temporal lobe and frontal cortex also participate in face recognition and perception tasks. (2) Each structure has a distinct "response profile"; that is, with respect to a given task certain structures respond strongly, others less or not at all. This profile might change with a different task, although the physical parameters of the stimuli remain the same. (3) The right hemispheric predominance of face processing, as suggested by patient data and studies in healthy volunteers, seemed to be restricted to its early stages (i.e., before 100-150 ms). (4) Recognition of faces might be associated with differential intracranial responses, despite an incorrect overt response, reflecting neurophysiological correlates of implicit memory. (5) The more the stimulus resembled a complete human face, the earlier and larger the N200 response was found, in particular over the basal temporobasal cortex. Analysis of electrical signals from intracranial electrodes might help to improve our understanding of the underlying physiological and anatomical constraints of cognitive processes. 2001 Elsevier Science
\end{abstract}

Face recognition, one of the first higher-order visual processes to develop in humans, is already observed in 3-day-old human neonates (1). Successful face perception, recognition of face identity, and correct interpretation of facial expressions are mandatory for proper social functioning. Bodamer was the first to describe the failure to recognize familiar persons by their face (2) (as compared with nonface visual stim-

\footnotetext{
${ }^{1}$ To whom correspondence should be addressed at 330 Brookline Avenue, EEG Laboratory, GZ-5, Boston, MA 02215.
}

uli) and called this disorder "prosopagnosia." Subsequently interest in disturbed face recognition increased and led to several reports of similar deficits. Damasio et al. reviewed these reports and found that, in humans, the most crucial structures for face recognition are localized bilaterally in the temporo-occipital junction, including the fusiform and lingual gyri (3). However, tachistoscopic studies in healthy volunteers showed repeatedly that the right hemisphere plays a more crucial role in face perception/recognition (4). These results, as well as the observation of face recog- 
nition by the neonate (which also includes recognition of faces other than the mother's (1)), add to other recent evidence obtained from imaging studies suggesting that the right hemisphere develops earlier than the left (5). Further evidence for a predominant role of the right hemisphere came from an autopsy case of a patient who suffered from prosopagnosia just prior to her death. Postmortem revealed a fresh infarction only in the right temporo-occipital cortex (6). The right hemispheric predominance for face recognition is not absolute and might change depending on task requirements (7).

After the anatomical correlates of face recognition were identified, interest arose in the electrophysiological correlates of this complex visual process as measured by scalp EEG in healthy volunteers and in patients with prosopagnosia. Cognitive evoked potential (EP) studies were carried out, i.e., EEG recordings triggered by the presentation of faces and control stimuli. The earliest studies, using only a few scalp electrodes, showed a large EP component at 170 to 200 ms that was related to faces but not to other stimuli (8-11). However, while these studies demonstrated relevant time epochs, the localization of these processes needs to be done using complementary techniques. Thus, over the last 10 to 20 years, positron emission tomography (PET) and functional MRI studies looked further into the anatomical correlates in vivo (12-14). While the underlying brain structures can now be precisely localized to within a precision of a few millimeters, no information about the temporal aspects of face processing was obtained using these techniques. This can be done by two other methods: the magnetoencephalogram (MEG) and the electroencephalogram (EEG). However, if based on routine scalp electrodes these techniques lack spatial resolution. Evoked potentials obtained from intracranial electrodes offer both an adequate time resolution in the millisecond range and, with the knowledge of electrode positions, also the precise localization of distinct EP components, reflecting underlying cognitive activity. As we will see in this review, cognitive processes, such as face perception and recognition, activate a large-scale network in which each participating brain structure has its specific performance profile.

\section{CATEGORY-RELATED PROCESSING: FACES VERSUS NONFACE STIMULI}

Invasive EP studies in patients who otherwise have no significant problem with face perception are par- ticularly useful for elucidating anatomical and functional correlates. In the paradigms used for this particular question, pictures of unknown faces were shown together with objects or abstract pattern. Differential responses to faces versus other nonface categories are then analyzed with respect to their latency and/or location.

Allison and co-workers studied neurophysiological correlates of face perception in 98 epileptic patients using subdural recordings over the extrastriate cortex $(15,16)$. Unknown faces, front views of cars, scrambled faces, and scrambled cars were used as visual stimuli. To enhance attention, photographs of butterflies were interspersed and the subject had to press a button whenever this stimulus appeared. Recordings were obtained from the basal and lateral aspects of the posterior temporal and occipital lobes of both hemispheres. From the left and right fusiform gyri, more rarely from the inferior temporal gyrus, a large peak between 150 and $200 \mathrm{~ms}$ (N200) was evoked by faces but not by the other stimuli. This latency is in agreement with the face-specific components measured in scalp studies (8-11). Additional components (P150, P290, N700) were markedly less face-specific ( $\mathrm{N}$ and $\mathrm{P}$ indicate polarity and the number is the average latency in milliseconds).

The localization in the basal temporo-occipital cortex and the fact that the components were obtained from both hemispheres are concordant with previous lesion studies (3). Some of these patients were studied with color stimuli, i.e., blue and red checkerboards on which the subject had to indicate if the colors were the same or not (17). It was shown that the "face region" is somewhat anterior to the "color region," which was found in the posterior fusiform gyrus. No overlap of these two regions was observed in any patient, although both were close. This confirms the clinical observation of the often-noted coexistence of prosopagnosia and achromatopsia in cases of a vascular accident in this part of the brain (4). A few sites over the lateroposterior temporal cortex (left and right hemispheres) showed a face-related N200 as well as the remaining components (16). There were anatomically separate from the ventral N200 site. Interestingly, the latencies of the P150 and N200 occurred earlier over the lateral than the basal sites.

The neurophysiological correlates of face preception and their anatomical location were investigated in a study of a total of 33 patients with depth electrodes $(18,19)$. The subjects had to indicate by a pushbutton response if the face has been repeated or not. Ten faces were repeated 12 times and the associated responses 
were compared with those of 120 novel faces. As a control task, the same paradigm was presented with four- to seven-letter words. Although the neurophysiological correlates of the mnestic aspects of face processing were addressed (discussed below), overall differences of responses to faces and words were analyzed as well. Results were described separately for posterior (18) and frontal (19) recordings sites. Eight distinct components were found in the posterior cortex of their patients: N75-P105, N130-P180-N240, N310-N430, P630. Interestingly, the first component complex (N75-105 ms) was evoked primarily by faces and localized in the lingual gyrus. Of the following component complex, the P180 was even more specific to faces and located in the fusiform gyrus. This component might correspond to the "face-specific" potential at a latency of 170 to $200 \mathrm{~ms}$ described in several scalp studies (8-11). In comparison, word-specific components did not start before $190 \mathrm{~ms}$ and correlated to a potential sequence P190-P250 (instead of N130P180-N240). The last components occurring between 310 and $630 \mathrm{~ms}$ were noted in several brain areas, maximally in the hippocampus. These components revealed only little category specificity.

In the frontal and central areas (19), a triphasic waveform N120-P170-N220 is evoked by faces, resembling the posterior N130-P180-N240 complex. The amplitudes are larger than those found for words, so it was conjectured that these responses are facespecific. They are observed in the ventrolateral aspect of the prefrontal cortex and probably generated locally. This observation is in agreement with animal studies that found face-selective neurons in the ventral part of the frontal lobe of nonhuman primates (20). Taken together, face-related responses appear already at 120 ms outside the temporo-occipital cortex, where the first processing is carried out. The results suggest that face recognition involves a large-scale network, which leaves the striate and extrastriate cortex between 105 and 120 ms. Already at this short latency, the temporal, parietal, and frontal cortices are implicated. As in the previous study $(5,16)$, no major hemispheric predominance seemed to be effective for the EP components. That a multitude of brain regions participated in face processing was supported by a recent fMRI study (14).

In one patient (19), face-specific potentials were recorded at or near the motor face area. A link between perception of other people's faces and control of one's own face is suggested, as evident already in the newborn $(22,23)$.
The ventral processing stream, projecting from the occipital to temporal structures, is implicated in category-related perception, i.e., "what" of the visual stimulus (see above). As such, we explored more specifically the contribution of temporal structures to the differentiation between face and nonface stimuli and compared it with frontal lobe responses (24). Pairs of objects or faces were sequentially presented in patients with depth electrodes bilaterally in the frontal and temporal lobes. All faces were front view and unknown to the viewer. The objects were tools of daily use (e.g., toothbrush). The subject had to indicate with a pushbutton response if both faces or objects within the pair were the same or not. Regarding the category effect, differential responses to faces versus objects were analyzed. The "match effect" corresponds to EPs related to "same faces and objects" versus "different faces/objects." Four different anatomical regions of each subject were subjected to analysis: amygdala, hippocampus, temporal neocortex, and dorsolateral frontal cortex.

When comparing responses to face and object stimuli, differential EPs were found mainly in the mid- to inferior temporal neocortex, corresponding to a total of $59 \%$ of all relevant differential responses. Moreover, they were seen more often in the right than left hemisphere. However, responses were also seen in the hippocampus and dorsolateral frontal cortex. No response was noted in the amygdala. When responses related to matching versus nonmatching items were analyzed (for both stimulus categories), we found the same distribution. All subjects had such responses in the temporal neocortex ( $73 \%$ of all relevant responses) and some subjects also had responses in the hippocampus and dorsolateral frontal cortex, but none were found in the amygdala. Of the 17 sites where differential category responses were observed, 13 also displayed a "match effect." Thus, the category and perceptual match effects displayed a very similar anatomical distribution (Fig. 1).

With microelectrodes, Ojemann and collaborators recorded the activity of single neurons or neuronal units in the exposed right lateral temporal lobe. Neuronal activity was measured during several tasks involving face, object, and complex pattern matching as well as the labeling of facial emotional expressions (25). In all 11 patients, 21 neuronal populations were described that showed significant changes in neuronal activity in one of these tasks. One-third showed excitation whenever a face was shown, confirming animal studies that revealed neuronal responses specifically to faces in the superior and inferior temporal cortex 


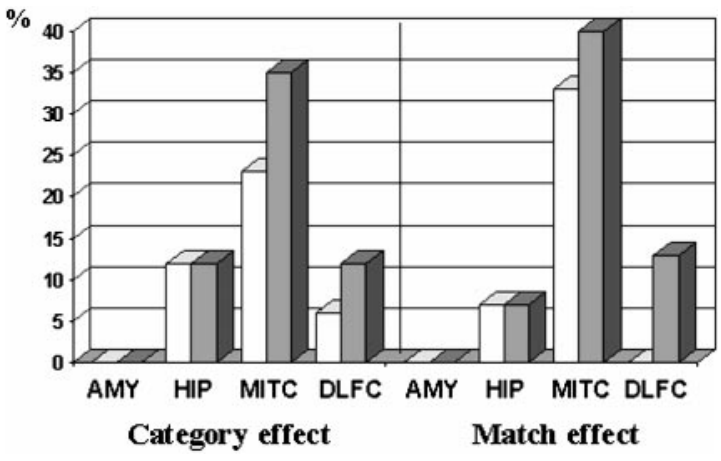

FIG. 1. Differential responses between faces and objects in four distinct anatomical sites. AMY, amygdala; HIP, hippocampus; MITC, mid- to inferior temporal neocortex; DLFC, dorsolateral frontal cortex. The same profile of the relative response frequency is observed for both effects (category: face vs object; Match: same vs different). Light bars: left side. Dark bars: right side. Note that most of the responses were obtained in the in midtemporal and inferotemporal cortex (accounting for 59 and $73 \%$ of all relevant differential responses. Adapted, with permission, from Seeck et al. (24).

$(26,27)$. Faces elicited the most frequent activity increase: $62 \%$ during a face matching task (of unknown faces), and $52 \%$ during the emotion labeling task. Only $38 \%$ of the units responded to complex figure matching or naming, respectively. A few neurons responded during matching per se, i.e., associated with both face and complex figure matching. Since no recordings from the left temporal lobe were reported, it is unclear if these findings represent a lateralized response pattern.

Another line of research made use of microelectrode recordings in patients with implanted electrodes. In these cases (e.g., (28)), the depth electrodes served as vehicle containing in their lumens small wires that allowed the recording of one or a few neurons at the end of the depth electrode. Since most studies were carried out in patients with electrodes implanted in both temporal lobes, conclusions can be drawn only from mesial structures such as the hippocampus, amygdala, and entorhinal cortex.

Fried and co-workers (28) recorded neuronal activity from these structures in nine patients. They described cells that differentiated between face and nonface stimuli, gender, and emotional expression or degree of familiarity of the face. Interestingly, in the entorhinal cortex and hippocampus, 37\% of the units responded only to certain conjunctions of gender, facial expression, and stimulus novelty. This finding provides evidence that the human hippocampus and functionally related structures are not merely a storage site, but are capable of extracting stimulus infor- mation and relating it to a situational context or further memory processing.

\section{PERCEPTION OF HUMAN FACES AS COMPARED WITH FACES FROM OTHER SPECIES}

Faces of some animal species have features similar to those of human faces and are more popular as pets. Dogs and cats are the most common pets, and this might be due to their resemblance to human faces. The fact that animal faces may be represented in distinct cortical areas is illustrated by the case report of Assal and co-workers: a farmer continued to have difficulties in recognizing his cows (by their heads/"faces") but was able to recognize human faces (29). In patients with subdural electrodes, faces of dogs and cats have been presented and the evoked responses compared with the responses evoked by human faces and scrambled faces (resembling abstract pattern) (16). Twentyeight sites (13 in the left hemisphere) showing differential responses were analyzed. Only the face-specific component at $200 \mathrm{~ms}$ (see above) revealed significant differences with respect to stimulus category. It was of significantly higher amplitude and shorter latency to human than to animal faces. This was true for the basal and laterotemporal recordings in both hemispheres. Comparisons with other species that have faces with a less consistent degree of "human resemblance" (e.g., reptiles, poultry) have not yet been performed.

\section{PERCEPTION OF HUMAN FACES AS COMPARED WITH FACE PARTS AND OTHER BODY PARTS, DIFFERENT GAZE, AND HEAD POSITION}

There is evidence that parts of faces, in particular the eyes, are scanned more frequently than other parts (e.g., the nose) (30). Whole faces or eyes, lips, nose, and face contours alone were shown to patients with subdural electrodes, yielding a total number of 45 ventral and 6 lateral temporal sites with differential responses. It was found that full faces evoked earlier and larger amplitudes of the N200 at all sites. The amplitude decreases and becomes later progressively in the order eyes/contours, lips, nose.

The positions of the head and eyes have been varied. Experimentally, only the N200 component 
seemed to change with respect to changes in the eye and head positions. While gaze did not significantly influence any of the EP components, head position changed the N200. It was larger and earlier when the head faced the viewer. An earlier N200 was found when the eyes faced the viewer as compared when the eyes were averted. With respect to eye and head positions, a right hemispheric predominance of differential responses was noted (16).

Since in the monkey temporal lobe, not only facebut also hand-responsive cells have been reported (31, 32 ), pictures of hands, faces, and objects were presented to the same patients with subdural electrodes (16). Over the sites where face-specific responses were expected, hands did not induce significant differences. However, hands elicited "hand-specific" components (P165-N230-P310) which were recorded at different sites. While there were no hemispheric differences for hand EPs recorded from the basal cortex, a left predominance was seen over the lateral and posterior aspects of the temporal lobe. The latter finding is concordant with PET studies that stress the implication of left temporoparietal regions in the perception of biological motion (33). Moreover, patients with lesions in this region may suffer not only from aphasia, but also from ideomotor or ideatory apraxia, suggesting that this region is critical for the meaningful use of one or both hands.

\section{RECOGNITION OF PREVIOUSLY KNOWN FACES (FACE MEMORY)}

A very important social capacity is the ability to recognize previously seen people. This encompasses several aspects: (a) the recognition of highly familiar faces such as mother and father, (b) the recognition of celebrities who are highly familiar due to repetitive visual exposure but lack the direct encounter and the associated emotional impact, (c) recently seen faces who are otherwise unknown to the viewer. In the context of psychological or neurophysiological paradigms, the last variant is used most often when investigating mnestic face processes.

\section{Recognition of Highly Familiar Faces Personally Known by the Viewer}

Parts of the temporal lobe such as the amygdala and association cortex play a major role in memory function and emotional labeling that both come into the act

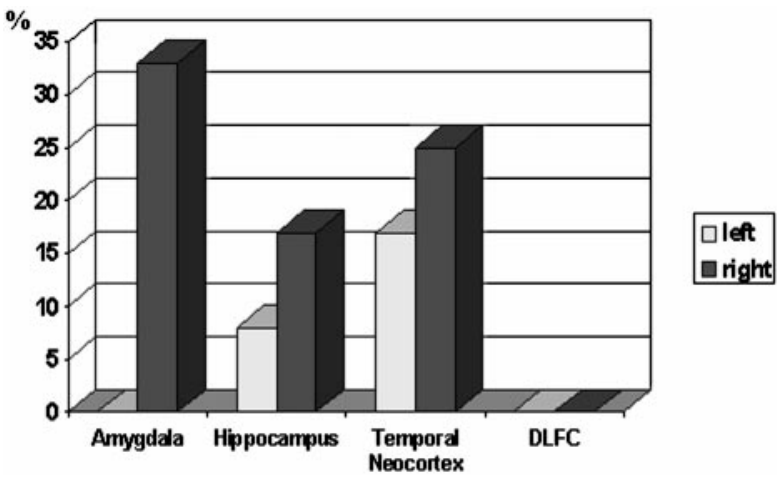

FIG. 2. Relative frequency of differential responses to very familiar faces in the amygdala, hippocampus, lateral temporal neocortex (mid- to inferior temporal gyrus), and dorsolateral frontal cortex (DLFC). Note the relative high frequency of responses in the right amygdala and their absence in the frontal cortex. More right and left hemispheric responses were obtained. Adapted, with permission, from Seeck et al. (35).

of recognizing a close friend or family member (34). To investigate this type of face recognition, photographs of family members and close friends, as well as unknown faces with similar facial expressions, were presented to patients with epilepsy who had depth electrodes in the frontal and temporal lobes (35).

In all patients, prominent differences between familiar and unfamiliar faces were found in mesial and lateral temporal lobe recordings, but not in frontal lobe recordings. The right amygdala was most frequently associated with differential responses. Overall, a right hemispheric predominance was noted (Fig. 2).

These results suggest that the temporal lobe, in particular the right amygdala, is specialized for recognizing experiential attributes such as familiarity. The encoding of "familiarity" by structures of the medial temporal lobe is consistent with phenomena such as "déjà vu/véçu" that are frequently reported by epileptic patients as part of their seizure semiology or after electrical stimulation of medial temporal lobe structures. The relatively high association of this response is consistent with neuropsychological evidence that the right hemisphere is more closely involved in face recognition $(6,36)$. The relatively high percentage of differential responses in the amygdala corroborates animal studies that attribute a critical role in associating complex visual information with emotional states $(37,38)$. Recognition of familiarity also entails the retrieval of visual long-term memory. The hippocampus and the inferotemporal cortex are crucial for this function, which explains why these structures were 
engaged by the familiar face recognition task whereas lateral frontal cortex was not.

\section{Recognition of Famous Faces}

In a report by Puce and co-workers, famous faces and unfamiliar faces, matched for sex, age, and attractiveness, were presented to patients implanted with subdural electrodes (39). Potentials were obtained from a wide array of electrodes over temporo-occipital and lateral temporal sites. Both stimulus categories were presented together with dog faces, which served as targets. A negative peak at $200 \mathrm{~ms}$ (N200), identified as the most face-specific component in this brain region, did not show any difference between famous and nonfamous faces. None of the later components (up to $700 \mathrm{~ms}$ ) differentiated between both categories in any of the recorded sites. Thus, brain regions that reveal face-specific responses do not necessarily differentiate within different face categories. The distinction between famous and unknown faces seems to need the recruitment of semantic information which is localized elsewhere. However, since the number of correctly identified famous faces is not given (i.e., the overall performance score in this task), it is possible that the lack of differences is due to an impaired knowledge of famous faces.

\section{Recognition of Previously Presented Faces Unknown to the Viewer}

Recognition of previously unknown faces is a fundamental capacity for social interaction and survival and present already in the newborn (1). Where and when a previously unknown face becomes known or even familiar has been the subject of several studies.

In the studies of Halgren and co-workers $(18,19)$ (discussed above) the consecutive stages of face recognition and their anatomical location were investigated in patients with depth electrodes. Face and word repetition paradigms were presented as outlined above. Discrete changes related to face repetition were observed after $130 \mathrm{~ms}$, localized in the lingual gyrus. The relatively face-specific component around $180 \mathrm{~ms}$ seemed to be unchanged; however, after $240 \mathrm{~ms}$ the identified components showed amplitude decrease at various sites in posterior and frontal cortices when repeated faces were presented.

In a study using subdural electrodes, a paired-associate paradigm was applied (39). In the learning period, the subject had to learn 10 unfamiliar names paired with fictional names. After a distractor phase, during which 10 new faces had to be labeled as male or female, the subject reviewed the 10 learned faces and indicated by a pushbutton response if the associated name was correct (50\% were correct). Among the components associated with face perception (N200, P290, N700, see also above), only P290 and N700 revealed changes related to repetition. The amplitude of P290 was significantly larger during the learning period and the amplitude of N700 was significantly larger during the identification phase. The authors concluded that there are "face recognition units" and "matching templates" as proposed elsewhere $(3,40)$, both occurring at temporally distinct processing levels. However, it should be noted that this paradigm confounds face and name recognition, although the retrieval of face-name pairs may be intimately associated with face recognition.

Intracranial microelectrode studies investigated face recognition of previously unknown faces as well (41, 42). In 14 patients with depth electrodes, neuronal activity and evoked potentials were recorded during recognition memory paradigms with words and faces. With respect to the face paradigm, the subject had to manually respond to the repeated stimulus. Thirty neuronal units were found that preferentially fired after the repetition of individual faces or words, but not to the face category as a whole. They were preferably found in the anterior hippocampus, showing maximal firing rate between 300 and 500 ms. In 11 of these 30 units, there was also increased excitation related to the motor response itself. Interestingly, one visual sensory unit, located in the posterior hippocampus, had an extremely short onset latency of less than $50 \mathrm{~ms}$. Overall, no responses associated with recognition per se of previously seen faces were noted in mesial temporal structures.

\section{IMPLICIT MEMORY OF FACES}

Recognition is not necessarily a conscious experience. This has been investigated in seven patients with bilateral depth electrodes in the frontal and temporal lobes (43). Pairs of unknown faces were presented some of which contained a repetition of a previous face. The subject had to indicate with a pushbutton response if one of the faces had already been presented or not. No face was repeated more than once. The subjects found the task difficult, so the performance showed considerable intersubject variability. On average, the subjects performed at chance level 


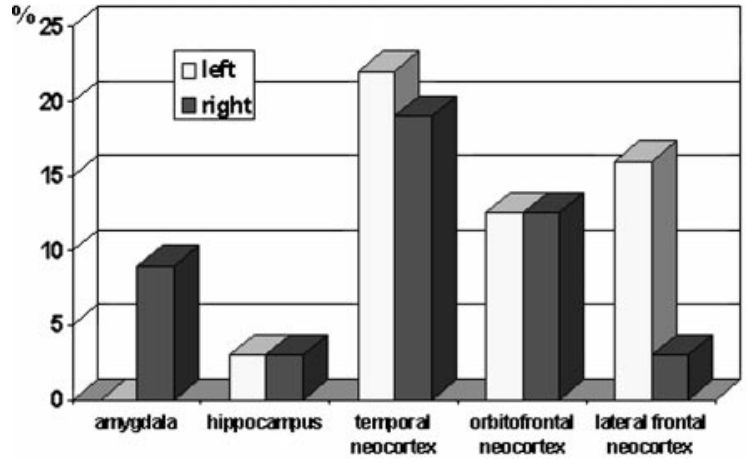

FIG. 3. Intracranial responses differentiating between repeated and novel faces, despite impaired overt performance, representing neurophysiological correlates of implicit knowledge. Note the predominance of responses in neocortical structures, in particular the temporal neocortex. Adapted, with permission, from Seeck et al. (43).

although they accomplished almost perfectly a simpler face-matching task (35).

Despite their inability to discriminate between repeated and nonrepeated faces in the behavioral task, all subjects showed differential visual EPs to the two types of stimuli.

Differential responses were more frequent in neocortical structures, such as the mid-inferotemporal cortex, the orbitofrontal cortex, and the lateral frontal cortex, than in limbic sites (amygdala, hippocampus). Among the neocortical sites, the mid- and inferotemporal cortex yielded most of the differential responses without consistent hemispheric predominance. However, in the amygdala, differential EPs were obtained more from the right than the left, and in the lateral frontal cortex, more from the left than the right hemisphere (Fig. 3).

This study has two principal findings. First, subjects who were unable to distinguish repeated from nonrepeated faces in an explicit behavioral task displayed differential EPs to these stimuli, demonstrating implicit encoding of the distinction between the two. Second, the differential responses were seen much more frequently in the temporal neocortex than in the limbic leads.

The recent literature has emphasized the existence of clear distinctions between "explicit" and "implicit" knowledge. Explicit knowledge leads to the intentional, declarative, and conscious awareness of recently experienced events. Implicit memory refers to priming or nondeclarative memory, and represents a nonconscious influence of past experience on current behavior, without the necessity of conscious recall of the prior exposure. The existence of implicit memory was repeatedly shown in several studies with amnesic patients and patients with face recognition deficits (44, 45). The amnesic syndrome, in which patients have severe deficits of explicit memory but relative preservation of implicit memory, is seen when the medial temporal lobes and diencephalon are damaged (46, $47)$, suggesting that these areas are not crucial for implicit memory. This notion is supported by our findings as well as by imaging studies (48-50). Further support comes from single-unit studies in nonhuman primates showing that the inferotemporal neurons give differential responses to novel versus previously seen objects $(51,52)$. In a microelectrode study in humans, neurons were found that responded to previous stimulus exposure but were unrelated to the subject's recollection (28). Taken together, these observations are consistent with current concepts $(46,53)$ that link implicit encoding to posterior unimodal association cortices. With respect to implicit face memory, the anatomic substrate is provided by the midand inferotemporal cortex. In certain circumstances, this process cannot access the neural circuits that lead to conscious awareness. This dissociation between implicit and explicit memory adds further evidence for the emerging view that consciousness is not a given by-product of cortical activity.

\section{WORKING MEMORY FOR FACES}

Apart from the distinction between implicit and explicit, memory can be defined along the duration or longevity of mnestic traces. Working memory is the type of memory that has limited capacities and is active or relevant only for a short period. Its duration is considered to be in the range of seconds ("scratchpad memory") and is distinguished from associative memory, which remains stable over time. The neurophysiological correlates of working memory for faces were investigated by comparing the responses to faces in two different paradigms. In the first paradigm, the patients had to decide if the face was familiar or not (24). This was an immediate decision and did not need to be put on hold. In the other paradigm the patients were asked to decide if two consecutive faces were the same or not. In that case, the first face had to be briefly memorized to be able to compare it with the second face (43). Thus response differences between these face categories should reflect differences in the working memory load. Both paradigms were carried out in the same patients, and the responses from four anatomical 


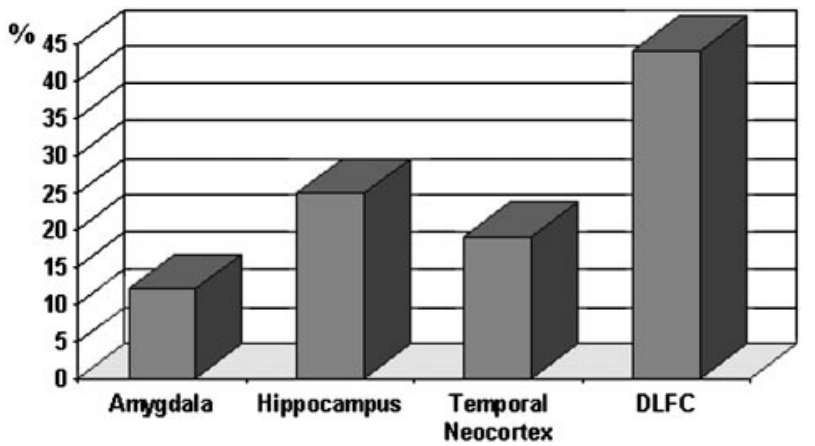

FIG. 4. Relative frequency of differential responses in the analyzed sites. DLFC, dorsolateral frontal cortex. Note that the almost half of the responses were found in the frontal cortex. Adapted, with permission, from Seeck et al. (24).

sites were analyzed (amygdala, hippocampus, midto inferior temporal neocortex, dorsolateral frontal cortex).

The working memory effect was found in all four structures analyzed; however, it was not uniquely distributed. Lateral frontal cortex and, to a lesser extent, the hippocampus displayed differential response patterns most frequently when the task that required the very brief on-line storage of information in working memory was compared with the task that had no such requirement (Fig. 4). No hemispheric differences were seen. There is anatomical and physiological evidence that the prefrontal cortex plays a pivotal role in working memory processes $(54,55)$. Our results are in line with this notion. The dorsolateral frontal cortex has rich connections with the hippocampal formation and adjacent cortex; most of these connections are bidirectional (54). This observation, obtained in nonhuman primates, might explain the relatively frequent responses also in the latter structure.

\section{THE SPEED OF FACE RECOGNITION}

The studies outlined above described intracerebral correlates of face or object perception as well as different types of face memory. These findings were corroborated by a large body of microelectrode studies in monkeys that revealed neurons in the superior or inferior temporal cortex that responded selectively to a certain stimulus category, such as faces or recognition of previously seen items $(37,38,56,57)$. These studies, together with findings from human scalp studies (58), consistently described differential effects during visual recognition tasks between 250 and 600 ms, occasionally as early as $150 \mathrm{~ms}$ (59). On the other hand there is a growing body of evidence that visual processing occurs faster than previously thought. Several human depth studies $(18,19,60,61)$, and microelectrode studies in humans (41) and monkeys (for review see 62) showed increased neuronal activity at $30-50 \mathrm{~ms}$ in the striate and extrastriate visual cortex as well as in the temporal lobe after onset of simple visual stimuli such as flashes, pattern reversal, and moving pattern. Since evidence accumulates that visual analysis is computed in a parallel rather than in a serial fashion, there is reason to believe that recognition memory of complex visual pattern starts before $100 \mathrm{~ms}$.

The same paradigm as described in (43) was presented to 12 male volunteers (group I). Moreover; the EPs obtained from patients with depth electrodes (43) (group II) were reanalyzed with special reference to early components (63). While the patients performed only at chance level, the normal subjects found the task also relatively difficult although the error rate was lower (about 6\%). We analyzed the data with particular emphasis on the period between 0 and $150 \mathrm{~ms}$.

EEG data were obtained from 13 electrodes, attached on the scalp according to the 10/20 system. With respect to group I, averages for each subject and category were calculated and compared statistically across subjects. Of these visual EPs, epochs of $20 \mathrm{~ms}$ were determined and the mean voltages within each epoch were analyzed statistically across subjects. With respect to group II, intracranial visual EPs were obtained and analyzed individually as described above.

In group I, differential responses between repeated and novel faces were seen as early as $50 \mathrm{~ms}$, with a trend already present between 30 and $50 \mathrm{~ms}$. The following intervals between 90 and $190 \mathrm{~ms}$ did not show significant differences between novel and repeated faces. Between 190 and 600 ms, the visual EPs of the healthy subjects revealed almost continuously highly significant effects, which is consistent with previous studies (64).

In all subjects of group II, early responses $(<110 \mathrm{~ms})$ were also found, although overt performance differed from that of the control group (Fig. 5). The responses were independent of the site of the epileptogenic focus. While late responses were distributed evenly in both hemispheres, the early responses were obtained twice as often in the right hemisphere $(P=0.0006)$. Most of the differential visual EPs were found in the mid- and inferior temporal cortex $(46 \%$ of all responses, $P<0.001)$. 


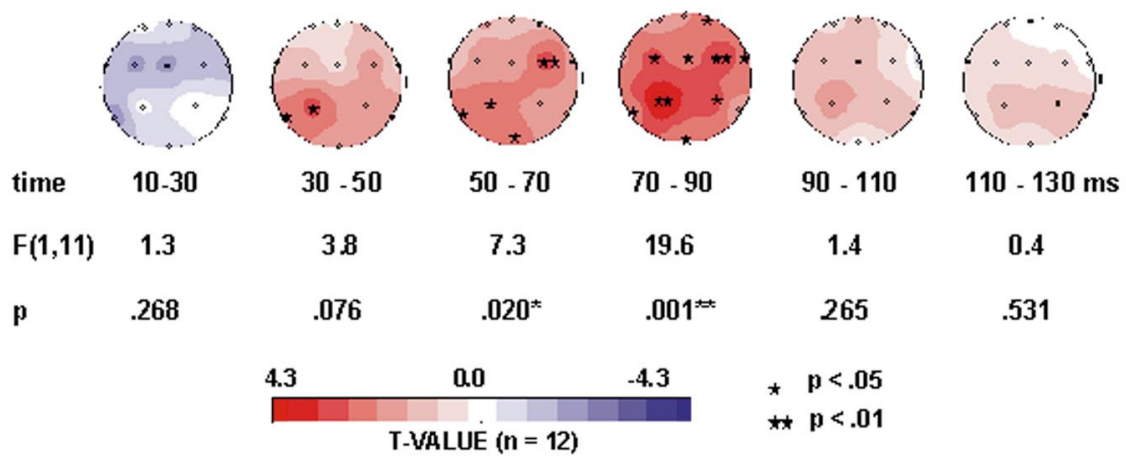

FIG. 5. Topographic maps (nose up, left ear left) of the statistical comparisons of the voltages over 20-ms epochs between repeated and nonrepeated faces in group II. *Electrodes with $P<0.05$; ${ }^{* *}$ electrodes with $P<0.01$. The results for the early differential responses between 10 and $130 \mathrm{~ms}$ are shown. Below the maps, the results of global tests using $13 \times 2$ repeated-measure analysis of variances (ANOVAs) are given for each 20-ms epoch. The $F$ and $P$ values for the condition factor are given. Significant differences were found between 50 and 90 ms, with a trend between 30 and 50 ms. Reprinted, with permission, from Seeck et al. (63).

We concluded that face recognition processes are extremely fast and already present at 50-70 ms, confirming two earlier scalp studies that found differential responses between 45 and $60 \mathrm{~ms}(65,66)$. Analysis of neuronal responses with information theory methods indicated that sufficient visual information can be transmitted within $30 \mathrm{~ms}$ (67). Moreover, the early components are modulated in patients with left or right temporal lobectomy, indicating that their presence relies on the integrity of the temporal lobe structures (66).

\section{CONCLUSION}

Face perception and recognition is an intriguing capacity, present since birth, that constitutes an essential part of social perception in general. Efficient face processing is vital for social survival, and is not restricted to our species. From studies in monkeys, careful patient observations, and imaging studies, the anatomical correlates of this complex visual task are known. The approaches that have been used to assess the anatomical and physiological study of face perception may serve as a model to study the neurophysiological aspects of other higher cognitive processing and may give some insight into cerebral organization.

Several findings appear important in the frame of this review. First, complex visual processing, such as face perception and recognition, involves a distributed but regionally specialized neuronal network. The same brain regions, or more specifically the same electrode contacts, show differential responses to a variety of tasks but not in a uniform fashion. For example, the differentiation of faces from other nonface stimuli seemed to be carried out primarily in the lingual and fusiform gyrus of the basal temporo-occipital cortex and/or in the lateral temporal neocortex, but less frequently in the amygdala. The reverse is true when a close family member has to be recognized on a photo. If novel faces have to be discriminated from repeated, otherwise unknown faces, the lateral temporal and, to a lesser degree, also the lateral frontal and frontoorbital cortex but not the basal temporo-occipital cortex become active. Other structures are less important for primary visual processing. While the frontal lobe differentiates somewhat between faces and nonfaces, it seems that it does not contribute to identification of the family member. However, if the working memory aspect becomes relevant, the frontal lobe contribution increases. The analysis of the relative frequency of differential responses with respect to the recorded brain regions is shown in Fig. 6 . The distinct profile of the "specialization" of each brain region represents an efficient and economic way of functioning.

Concerning the lateralization of differential responses between face and nonface stimuli, the intracranial studies suggest a right hemispheric predominance in lateral temporal cortex rather than in the fusiform/lingual gyrus. This may corroborate the observation of Damasio and co-workers and recent imaging studies that did not find evidence of a clear-cut lateralization in patients with lesions in the fusiform gyrus $(3,68)$. It may suggest that the lateral extrastriate cortex is more of a higher-order visual association cortex than the basal temporo-occipital junction. It is only within the hierarchically higher cortex, where the right hemisphere predominance for nonverbal pro- 

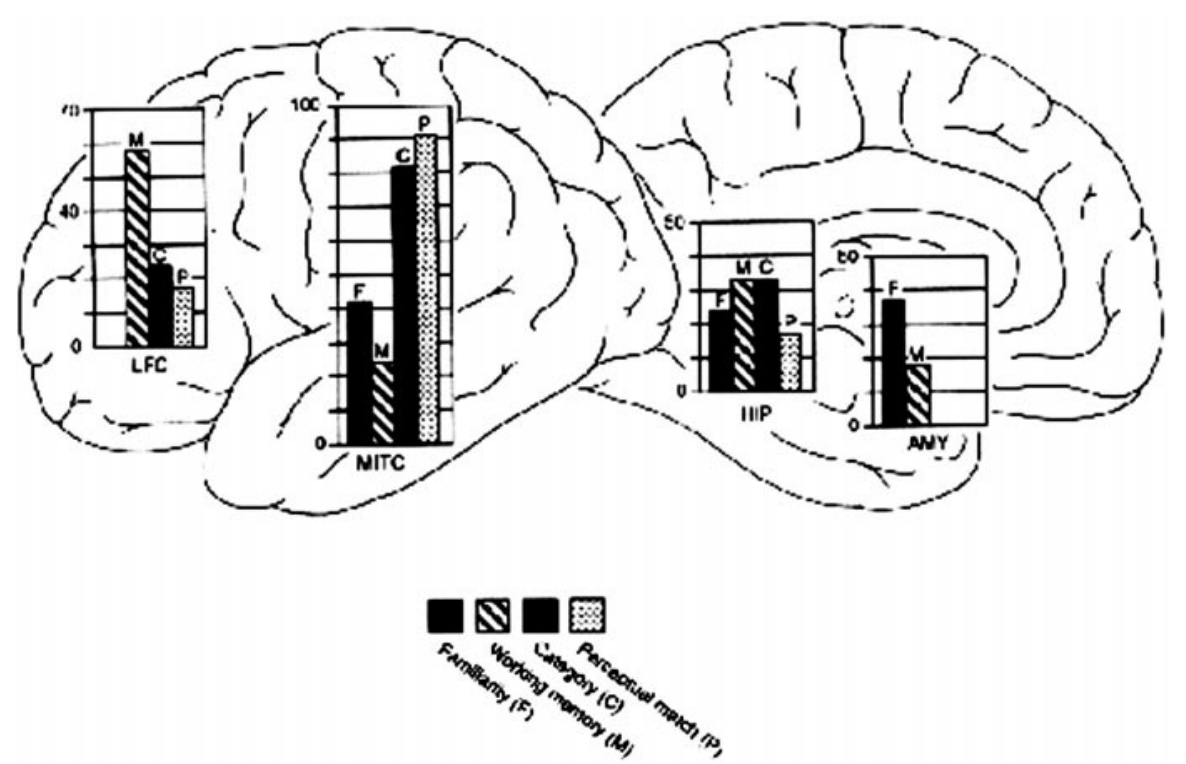

FIG. 6. Regional variations in the frequency (indicated on the $y$ axis) with which different parts of the brain were engaged in the four types of visual encoding that were investigated. AMY, amygdala; HIP, hippocampus; LFC, lateral frontal cortex; MITC, midtemporal and inferotemporal cortex. Reprinted, with permission, from Seeck et al. (24).

cessing becomes active. In other words, even within the visual association cortex, an internal hierarchy exists with more or less lateralized contribution. The unraveling of various degrees of hemispheric specialization within distinct steps of cognitive processing seemed to be best elucidated with neurophysiological techniques.

Most studies agree that face processing is more rapid than previously thought. Halgren and co-workers as well as our own studies indicate that already at 50-70 ms, differential responses between face and nonface stimuli or between novel and repeated faces occur. Human microelectrode studies found neuronal responses in the range of $50 \mathrm{~ms}$. Between 100 and 120 $\mathrm{ms}$, face-related responses are already seen in the frontal cortex, consistent with our own observation that parts of the frontal cortex (i.e., the frontal eye field) participate in visual processing around $100 \mathrm{~ms}$ (69). Interestingly, studies revealing early differential responses used depth rather than subdural electrodes. Whether this represents a chance finding or indicates that fast processing is carried out preferentially by neurons in the depth of the sulcus, less well recorded by subdural electrodes, is yet unknown.

Regarding the anatomical pathways of rapid face processing, several explanations emerge. Projections to extrastriate areas from the pulvinar or the lateral geniculate nucleus $(70,71)$ may become active and send a "copy" to the temporo-occipital cortex without passing through the striate cortex. This pathway may also be active in blind people showing the "blindsight" phenomenon (72). Alternatively, the normal pathway to visual association cortex via the striate cortex may work faster and more efficiently than previously thought. Since studies using subdural electrodes in basal temporo-occipital cortex did not show components earlier than $100 \mathrm{~ms}$ (17), this may speak in favor for the "short-cut" hypothesis. Moreover, as described in subdural electrode studies, face-specific responses between 150 and $200 \mathrm{~ms}$, anatomically distinct from the basal N200 site, occurred earlier over the lateral cortex (16). Because category identification (e.g., face vs flower) is also known to be carried out predominantly in the temporal neocortex, this brain region would harbor an efficient local network for face identification. Whether rapid processing is specific for face stimuli remains to be shown in future studies (73).

Hemispheric predominance seems not to be a steady state of mind but present at certain processing levels. Only early components of face recognition were significantly more often found in the right hemisphere. Late aspects of face processing are carried out in both hemispheres $(39,43)$, and during that stage the left hemisphere may be able to compensate a right hemispheric breakdown. However, since the right hemisphere treats face stimuli more rapidly and effi- 
ciently than the left hemisphere, it is the period up to $100 \mathrm{~ms}$ that is most relevant for this capacity.

Analyzing the latencies of differential responses might shed light on how the brain works. As outlined in several studies, perception of a repeated (vs novel) face elicits a differential response up to $90 \mathrm{~ms}$, then no difference until 190-200 ms, and then again up to $600 \mathrm{~ms}$ (18, $19,39,63)$. One could imagine that a rough analysis of the visual stimulus is carried out early in the processing. This could implicate mnestic templates, since the presence of early components relies on the integrity of the temporal lobes (66). However, scalp and intracranial EP studies consistently described differences between faces and nonface stimuli between 170 and $200 \mathrm{~ms}$ (8-11, 16, 17). Thus, a detailed analysis of the visual category is not the first stage of analysis, as one would imagine, but follows the rough analysis "item is known or unknown." Detailed analysis with respect to the task, i.e., reprise of the analysis "item is known or unknown" or "matches to previously shown item," is carried out after the category has been determined, during a relatively long period of several hundred milliseconds. These results are in line with the concept of "brain functional microstates" derived from the observation of lasting periods of stable configuration of the electric fields on the scalp surface $(74,75)$.

The contribution of microelectrode studies is difficult to appreciate. It seems that on the neuronal level (i.e., of one or several neurons), the responses are much more specific. As pointed out above, neurons responded to certain faces (but not to faces as perceptual category) or to certain conjunctions of face, gender, and age. It is only with EP studies that the differential responses along predefined categories are found (e.g., repeated vs novel). Thus, particularly the recording of neuronal activity from a larger brain region (corresponding to the "net activity" at a given electrode site) permits one to draw conclusions about the regional specialization.

When it comes to intracranial EP studies, the objection that the results are obtained in nonhealthy brains might be easily raised. However, the similarity of the latencies of the face-specific components in patients with intracranial electrodes and healthy volunteers with scalp electrodes suggests that both groups are comparable with respect to their functional organization.

\section{REFERENCES}

1. Pascalis O, de Schonen S. Recognition memory in 3- to 4-dayold human neonates. NeuroReport 1994;5:1721-4.
2. Bodamer J. Die Prosop-Agnosie (Die Agnosie des Physiognomie-Erkennens). Arch Psychiatr Nervenkr 1947;179:6-54.

3. Damasio AR, Damasio H, Van Hoesen GW. Prosopagnosia: anatomic basis and behavioral mechanisms. Neurology 1982; 32:331-41.

4. Grüsser OJ, Landis T. Man as a social partner in the visual world: perception and recognition of faces and facial expression. In: Cronly-Dillon JR, editor. Vision and visual dysfunction, vol 12: Visual Agnosia and other disturbances of visual perception and cognition, New York: Macmillan Press, 1999. 218-39.

5. Landis T, Regard M, Bliestle A, Kleihues P. Prosopagnosia and agnosia for noncanonical views: an autopsied case. Brain 1988; 111:1287-97.

6. Landis T, Regard M, Bliestle A, Kleihus P. Prosopagnosia and agnosia for noncanonical views. Brain 1988;111:1287-97.

7. Marzi CA, Tassinari G, Tressoldi PE, Barry C, Grabowska A. Hemispheric asymmetry in face perception tasks of different cognitive requirement. Hum Neurobiol 1985;4:15-20.

8. Small M. Asymmetrical evoked potentials in response to face stimuli. Cortex 1983;19:441-50.

9. Botzel K, Grusser OJ. Electric brain potentials evoked by pictures of faces and non-faces: a search for "face-specific" EEG potentials. Exp Brain Res 1989;77:349-60.

10. Jeffreys DA, Tukmachi ES. The vertex-positive scalp potential evoked by faces and by objects. Exp Brain Res 1992;91:340-50.

11. Seeck M, Grüsser OJ. Category-related components in visual evoked potentials: photographs of faces, persons, flowers and tools as stimuli. Exp Brain Res 1992;92:338-49.

12. Nakamura K, Kawashima R, Sato N, Nakamura A, Sugiura M, Kato T, Hatano K, Ito K, Fukuda H, Schormann T, Zilles K. Functional delineation of the human occipito-temporal areas related to face and scene processing: a PET study. Brain 2000; 123:1903-12.

13. Cabeza R, Nyberg L. Imaging cognition. II. An empirical review of 275 PET and MRI studies. J Cogn Neurosci 2000;12:147.

14. Leveroni CL, Seidenberg M, Mayer AR, Mead LA, Binder JR, Rao SM. Neural systems underlying the recognition of familiar and newly learned faces. J Neurosci 2000;20:878-86.

15. Allison T, Ginter H, McCarthy G, Nobre AC, Puce A, Luby M, Spencer DD. Face recognition in human extrastriate cortex. J Neurophysiol 1994;71:821-5.

16. Allison T, Puce A, Spencer DD, McCarthy G. Electrophysiological studies of human face perception. I. Potentials generated in occipitotemporal cortex by face and non-face stimuli. Cerebral Cortex 1999;9:415-30.

17. Allison T, Begleiter A, McCarthy G, Roessler E, Nobre AC, Spencer DD. Electrophysiological studies of color processing in human visual cortex. EEG Clin Neurophysiol 1994;88:343-55.

18. Halgren E, Baudena P, Heit G, Clarke JM, Marinkovic K. Spatio-temporal stages in face and word processing. I. Depthrecorded potentials in the human occipital, temporal and parietal lobes. J Physiol (Paris) 1994;88:1-50.

19. Halgren E, Baudena P, Heit G, Clarke JM, Marinkovic K, Chauvel P. Spatio-temporal stages in face and word processing. 2. Depth-recorded potentials in the human frontal and Rolandic cortices. J Physiol (Paris) 1994;88:51-80.

20. Oscalaidhe SP, Wilson FA, Goldman-Rakic PS. Areal segregation of face-processing neurons in prefrontal cortex. Science 1997;278:1135-8.

21. Deleted in proof. 
22. Meltzoff AN, Moore MK. Imitation of facial and manual gestures by human neonates. Science 1977;198:75-8.

23. Field TM, Woodson R, Greenberg R, Cohen D. Discrimination and imitation of facial expressions by human neonates. Science 1982;218:179-81.

24. Seeck M, Schomer D, Mainwaring N, et al. Selectively distributed processing of visual object recognition in the temporal and frontal lobes of the human brain. Ann Neurol 1995;37:53845.

25. Ojemann JG, Ojemann GA, Lettich E. Neuronal activity related to faces and matching in human right nondominant temporal cortex. Brain 1992;115:1-13.

26. Perrett DI, Smith PAJ, Potter DD, et al. Neurones responsive to faces in the temporal cortex: studies of functional organization, sensitivity to identity and relation to perception. Hum Neurobiol 1984;3:197-208.

27. Rolls ET. Neurons in the cortex of the temporal lobe and in the amygdala of the monkey with responses selective for faces. Hum Neurobiol 1984;3:209-22.

28. Fried I, MacDonald KA, Wilson CL. Single neuron activity in human hippocampus and amygdala during recognition of faces and objects. Neuron 1997;18:753-65.

29. Assal G, Favre C, Anderes JP. Non-reconnaissance d'animaux familiers chez un paysan [Nonrecognition of familiar animals by a farmer: zooagnosia or prosopagnosia for animals]. Rev Neurol 1984;140:580-4.

30. Yarbus AL. Eye movements and vision. New York: Plenum, 1967.

31. Gross CG, Rocha-Miranda CE, Bender DB. Visual properties of neurons in inferior temporal cortex of the macaque. J Neurophysiol 1972;35:96-111.

32. Desimone R, Albright TD, Gross CG, Bruce C. Stimulus-selective properties of inferior temporal neurons in the macaque. J Neurosci 1984;4:2051-62.

33. Bonda E, Petrides M, Ostry D, Evans A. Specific involvement of human parietal systems and the amygdala in the perception of biological motion. J Neurosci 1996;16:3737-44.

34. LeDoux JE. Brain mechanisms of emotion and emotional learning. Curr Opin Neurobiol 1992;2:191-7.

35. Seeck M, Mainwaring N, Ives J, et al. Differential neural activity in the human temporal lobe evoked by faces of family members and friends. Ann Neurol 1993;34:369-72.

36. Sergent J, Ohta S, MacDonald B. Functional neuroanatomy of face and matching in human right nondominant temporal cortex. Brain 1992;115:15-36.

37. Leonard CM, Rolls ET, Wilson FAW, Baylis GC. Neurons in the amygdala selective for faces. Behav Brain Res 1985;15:159-76.

38. Brothers L, Ring B, Kling A. Response of neurons in the macaque amygdala to complex social stimuli. Behav Brain Res 1990;41:199-213.

39. Puce A, Allison T, McCarthy G. Electrophysiological studies of human face perception. III. Effects of top-down processing on face-specific potentials. Cerebral Cortex 1999;9:445-58.

40. Bruce V, Young A. Understanding face recognition. Br J Psychol 1986;77:305-27.

41. Heit G, Smith ME, Halgren E. Neuronal activity in the human medial temporal lobe during recognition memory. Brain 1990; 113:1093-112.

42. Heit G, Smith ME, Halgren E. Neural encoding of individual words and faces by the human hippocampus and amygdala. Nature 1988;333:773-5.
43. Seeck M, Mainwaring N, Cosgrove R, Blume H, Dubuisson D, Mesulam MM, Schomer DL. Neurophysiologic correlates of implicit face memory in intracranial visual evoked potentials. Neurology 1997;49:1312-6.

44. Bauer RM. Autonomic recognition of names and faces in prosopagnosia. Neuropsychologia 1984;22:457-69.

45. Tranel D, Damasio AR. Autonomic recognition of familiar faces by prosopagnosics: evidence for knowledge without awareness. Neurology 1985;35:119-20.

46. Mesulam MM. Large-scale neurocognitive networks and distributed processing for attention, language, and memory. Ann Neurol 1990;28:597-613.

47. Milner B, Corkin S, Teuber HL. Further analysis of the hippocampal amnesic syndrome: fourteen year follow-up study of H.M. Neuropsychologia 1968;6:215-34.

48. Schacter D, Chiu CYP, Ochsner KN. Implicit memory: a selective review. Annu Rev Neurol 1993;16:159-82.

49. Squire LR, Ojemann JG, Miezin FM, Peterson SE, Videen TO, Raichle ME. Activation of the hippocampus in normal humans: a functional anatomical study of memory. Proc Natl Acad Sci USA 1992;89:1837-41.

50. Bruckner RL, Goodman J, Burock M, et al. Functional-anatomic correlates of object priming in humans revealed by rapid presentation event-related fMRI. Neuron 1998;20:285-96.

51. Brown MW, Wilson FA, Riches IP. Neuronal evidence that inferotemporal cortex is more important than hippocampus in certain processes underlying recognition memory. Brain Res 1987;409:158-62.

52. Miller EK, Li L, Desimone R. Activity of neurons in anterior temporal cortex during a short-term memory task. J Neurosci 1993;13:1460-78.

53. Mesulam MM. Neurocognitive networks and selectively distributed processing. Rev Neurol 1994;150:564-9.

54. Goldman-Rakic PS, Friedman HS. The circuitry of working memory revealed by anatomy and metabolic imaging. In: Levin HS, Eisenberg HM, Benton AL, editors. Frontal lobe function and dysfunction. New York: Oxford Univ Press, 1991: 72-91.

55. Rainer G, Asaad WF, Miller EK. Selective representation of relevant information by neurons in the prefrontal cortex. Nature 1998;393:577-9.

56. Perrett DI, Rolls ET, Caan W. Visual neurones responsive to faces in the monkey temporal cortex. Exp Brain Res 1982;47: 329-42.

57. Rolls ET. Neurons in the cortex of the temporal lobe and in the amygdala of the monkey with responsive selective for faces. Hum Neurobiol 1984;3:209-22.

58. Rugg MD, Roberts RC, Potter DD, Pickles CD, Nagy ME. Event-related potentials related to recognition memory: effects of unilateral temporal lobectomy and temporal lobe epilepsy. Brain 1991;114:2313-32.

59. Smith ME, Halgren E. Attenuation of a sustained visual processing negativity after lesions that include the inferotemporal cortex. Electroencephalogr Clin Neurophysiol 1988;70:366-9.

60. Wilson CL, Babb TL, Halgren E, Crandall PH. Visual receptive fields and response properties of neurons in human temporal lobe and visual pathways. Brain 1983;106:473-502.

61. Ducati A, Fava E, Motti ED. Neuronal generators of the visual evoked potentials: intracerebral recording in awake humans. EEG Clin Neurophysiol 1988;71:89-99. 
62. Nowak LG, Bullier J. The timing of information transfer in the visual system. In: Rockland et al., editors. Cerebral cortex, vol 12. New York: Plenum, 1997:205-41.

63. Seeck M, Michel CM, Mainwaring N, et al. Evidence for rapid face recognition from scalp and intracranial electrodes. NeuroReport 1997;8:2749-54.

64. Hertz S, Porjesz B, Begleiter H, Chorlian D. Event-related potentials to faces: the effects of priming and recognition. Electroencephalogr Clin Neurophysiol 1994;92:342-51.

65. Landis T, Lehmann D, Mita T, Skrandies W. Evoked potential correlates of figure and ground. Int J Psychophysiol 1984;1: 345-8.

66. Rougier MB, Guillem F, N'Kaoua B, Rougier A, LeRebeller MJ, Claverie B. Memory-induced subcortical modulation of early visual evoked activity in temporal lobectomy patients. NeuroOphthalmology 1994;14:103-11.

67. Tovee MJ, Rolls ET, Treves A, Bellis RP. Information encoding and the responses of single neurons in the primate temporal visual cortex. J Neurophysiol 1993;70:640-54.

68. Ishai A, Ungerleider L, Martin A, Schouten JL, Haxby JV. Distributed representation of objects in the human ventral visual pathway. Proc Natl Acad Sci USA 1999;96:9379-84.
69. Blanke O, Morand S, Thut M, Michel CM, Spinelli L, Landis T, Seeck M. Visual activity in the human frontal eye field. NeuroReport 1999;10:925-30.

70. Fries $\mathrm{W}$. The projection from the lateral geniculate nucleus to the prestriate cortex of the macaque monkey. Proc R Soc London B 1981;213:73-86.

71. Standage GP, Beneveto LA. The organisation of connections between the pulvinar and visual area MT in the macaque monkey. Brain Res 1983;262:288-94.

72. Stoerig P, Cowey A. Blindsight in man and monkey. Brain 1997;120:535-59.

73. Debruille JB, Guillem F, Renault B. ERPs and chronometry of face recognition: following-up Seeck et al. and George et al. NeuroReport 1998;9:3349-53.

74. Lehmann D. Principles of spatial analysis. In: Gevins AS, and Rémond A, editors. Handbook of electroencephalography and clinical neurophysiology, vol + : Methods of analysis of brain electrical and magnetic signals. Amsterdam: Elsevier, 1987: 309-54.

75. Michel CM, Seeck M, Landis T. Spatio-temporal dynamics of human cognition. News Physiol Sci 1999;14:206-14. 